\title{
A case of chronic granulocytopenia associated with vasculitis and amyloidosis
}

\author{
G. H. JENNINGS, A. J. LEVI, AND J. REEVE \\ From Edgware General Hospital, Edgware Road, Edgware, and Northwick Park Hospital, Watford Road, \\ Harrow, Middlesex
}

SYNOPSIS A patient is described with chronic neutropenia who was followed for 22 years. She had varying patterns of neutropenia, sometimes cyclical, sometimes almost agranulocytic; at times the counts were normal. The course was complicated by a vasculitis and finally by large vessel thrombosis; extensive amyloid was found in small vessels at necropsy.

Neutropenia may occur in a variety of primary forms or be secondary to a number of drugs, poisons, and disease processes (Boggs and Wintrobe, 1970). Reimann (1963) described a number of patients with apparently primary disease who suffered from cyclic neutropenia and Morley, Carew, and Baikie (1967) described some further patients in whom the condition was familial. Other patients, however, suffer from a steady-state neutropenia, maintaining a low, stable neutrophil count for long periods. Among chronic disorders which have been described in association with neutropenia are systemic and discoid lupus erythematosus (Dubois, 1966).

This case is of interest for two reasons. First she was followed up for an exceptionally long period during which time she exhibited a variety of patterns of neutropenia. At times the neutropenia appeared to be cyclical, at other times the granulocyte counts were chronically depressed for prolonged periods, and there were even times when they were persistently elevated. Secondly, for a period during her illness she developed the manifestations of a collagen disorder, which responded rapidly to steroid therapy.

\section{Clinical Report}

The patient was first seen at the Edgware General Hospital in 1948, aged 13, with fever, pharyngitis, and enlarged cervical glands. The granulocyte count was found to be $100 / \mathrm{c} \mathrm{mm}$, other elements being normal. She gave a three-year history of similar brief episodes of fever, gingivitis, buccal ulceration, and coryza. A few days after admission to hospital,

Received for publication 12 June 1973. her symptoms and neutrophil count recovered. During regular observation by one of us.(G.H.J.) over the next 22 years, she at first followed a variable course with recurrent short attacks of buccal ulceration, of a similar nature, with a neutropenia occurring approximately once a month. There was also a liability to vulval ulceration and to superficial staphylococcal infections, and at times she developed almost total agranulocytosis, with which the recurrent staphylococcal infections were closely associated. She also had a history of hay fever in childhood.

The spleen became palpable in 1949 and was removed in 1951, but her clinical course remained unchanged. Histological examination of the spleen merely showed hyperplastic pulp reticulum. Bone marrow examinations in 1949 were normal twice but on a third occasion showed a marked reduction of the granular cells.

In 1953 she had an attack of erythema nodosum, following which her leucocyte count improved for a few months. Six years later (1959) she had the first of her allergic responses to one of many antibiotics. This was followed by a marked improvement in her leucocyte count (fig 1). She remained tolerant to penicillin, but over the following years developed an increasing range of allergies to various antibiotics.

In 1963, after a period of great emotional stress and mental disturbance, she became very weak and had a prolonged low-grade fever associated with neutropenia. The bone marrow appeared normal. There were muscular pains in the legs and thighs. On examination at this time, she had a faint macular rash over the body, a rash of butterfly distribution over the face, and marked muscle wasting and 


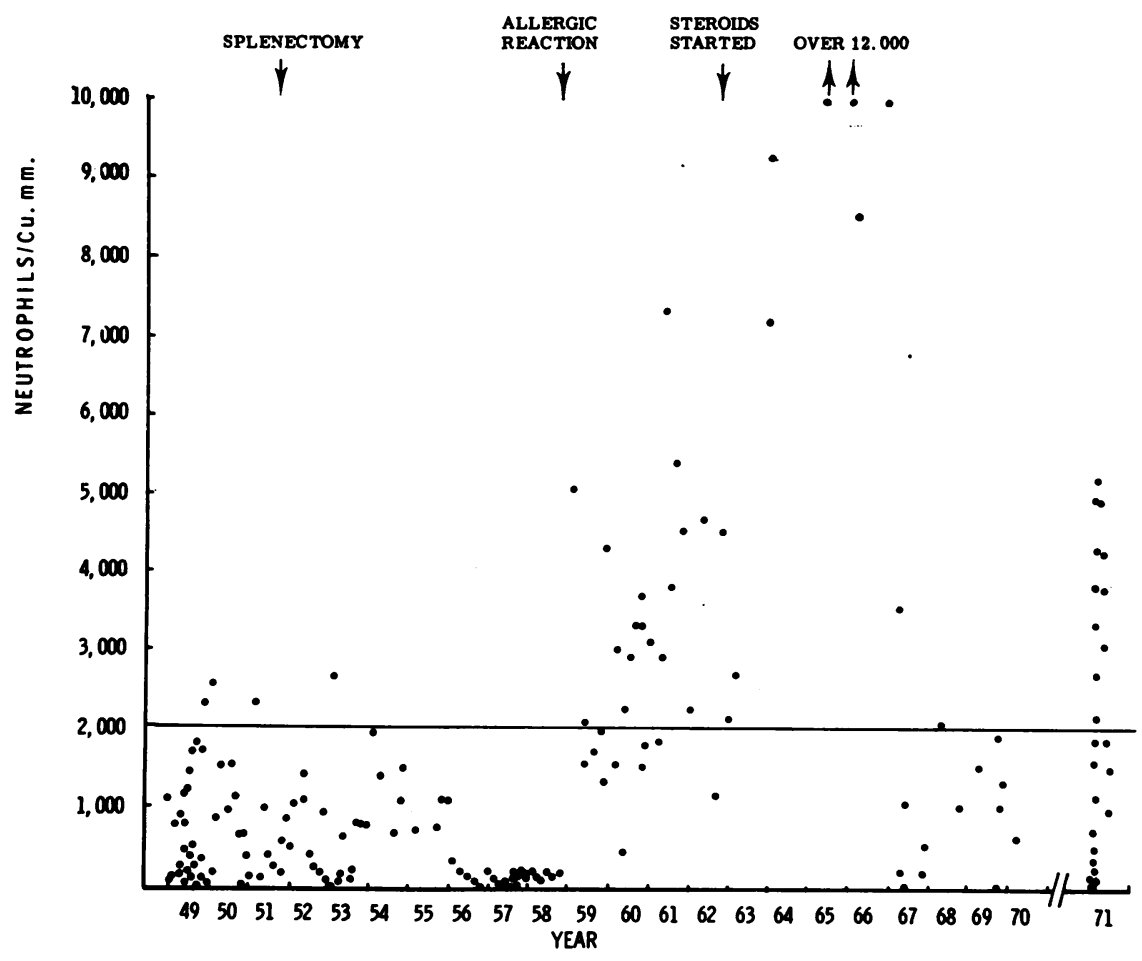

Fig 1 Neutrophil counts recorded since patient ${ }^{\circ}$ first presented in 1948.

weakness, particularly of the dorsiflexors of the left foot. Investigation failed to reveal an infective cause. The ESR (Westergren) was $23 \mathrm{~mm} / 1 \mathrm{hr}$, serum alpha 2 globulins were raised, but the antinuclear factor was negative, and no LE cells were seen in the blood. Biopsy of a small subcutaneous nodule from the left arm showed evidence of vasculitis (vide infra). On treatment with steroids, her neutrophil count rapidly rose to supranormal levels and her fever settled. Early reduction of the dosage resulted in immediate return of her symptoms and fever, but on a dose of $20 \mathrm{mg}$ of prednisone a day she rapidly gained strength and muscle bulk and there followed two years of good health with normal neutrophil counts. Fever and rheumatic pains in knees and thighs recurred twice in 1965. After 1966, she suffered from intermittent coldness and cyanosis of the hands and feet, associated with a recurrent indolent superficial ulceration of the ankles (fig 2). Peripheral pulses remained normal. In May 1967 she had severe cramp and coldness of the left hand, and the fingers of both hands were very stiff, with swelling of the interphalangeal joints. In 1969 she again lost much weight and showed brief agranulocytosis, fever, and severe mouth ulcers, as well as intense nervousness.

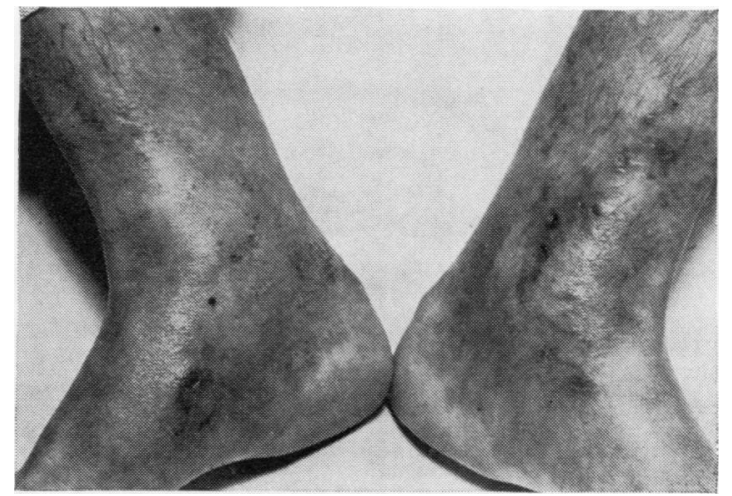

Fig 2 Photograph of patient's ankles in late 1966.

In January 1971, following a bath, both legs became cold and dead. The right one responded to extra warmth but the left did not, and she was admitted to Northwick Park Hospital. No peripheral pulses were palpable below the popliteal in the left leg and the foot was cold with reduced sensation.

Arteriography showed narrow vessels, but no block was demonstrated to below the level of the knee. Full conservative measures, including a 
marked increase in steroid therapy, failed to halt the progression of gangrene, and eventually below-knee amputation and lumbar sympathectomy were performed. In the late convalescent phase, while fully ambulant on crutches. she had a massive pulmonary embolus and died immediately.

\section{FAMILY HISTORY}

The patient's brother died in early manhood from acute nephritis, a matter of relevance in view of her later development of collagen disorder. She was thus left the sole support of an invalid mother, a situation causing much stress.

\section{PATHOLOGY}

A biopsy of a small subcutaneous nodule taken from the left arm in 1963, as already mentioned, showed vasculitis. The small artery illustrated (fig 3 ) showed recent thrombotic occlusion of the lumen. There was a heavy inflammatory cell infiltrate, maximal in the adventitia, but penetrating the full thickness of the vessel wall at one point.

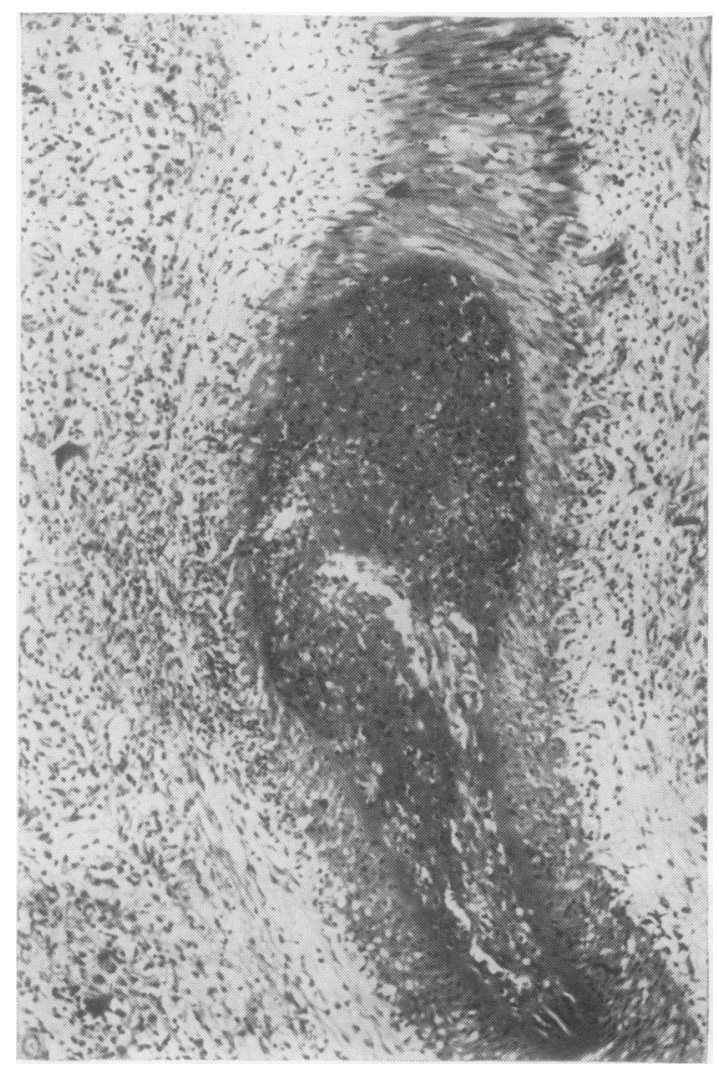

Fig 3 Histological section of biopsy of a small subcutaneous nodule taken from the left arm in $1963 \times 135$.

\section{POSTMORTEM FINDINGS}

\section{Gross}

Both pulmonary arteries were occluded by large recent emboli. No source was found in the systemic veins. The pericardium was bound down by adhesions. The left femoral artery was thrombosed and neither ulnar artery was patent. Otherwise no macroscopic abnormality was found.

\section{Microscopic}

Fibrous thickening of the pericardium was found and furthermore in the myocardium there were a few small foci of interstitial mononuclear cell infiltration. The femoral artery showed recent thrombus but no evidence of arteritis. No small vessel arteritis was found. Extensive amyloidosis confined to small arteries was found in the myocardium, kidneys, lungs, bone marrow, liver, and skin.

\section{Discussion}

During the 22-year follow up, this patient at times showed an apparent cyclical neutropenia, with periods of agranulocytosis, usually accompanied by ulceration, alternating with periods of good health with a normal white count. Subsequently, she developed a vasculitis as evidenced by the biopsy, but there was no evidence of this at necropsy after eight years' steroid treatment.

Neutropenias are frequently classified into 'cyclic' and 'non-cyclic' categories or according to severity. In the case of this patient, over a 22-year period, the neutropenia manifested itself in every degree of severity, at times approaching an apparently steady state, while at other times large variations in the neutrophil count suggested cycling. It has been suggested (Morley, 1967) that cyclic neutropenia is one stage in the progression to a severe degree of chronic steady-state neutropenia as the stem cells become less and less sensitive to the stimulus of neutropenia. In the case of this patient, episodes of profound neutropenia were always followed by partial recovery, sometimes spontaneous and at other times apparently triggered by steroid therapy or a drug reaction.

The association between chronic neutropenia of this duration and severity and other non-malignant chronic disorders is unusual. Neutropenia has been reported associated with agammaglobulinaemia, but in most cases is transient, associated with overt infection and recovery on treatment of the infection (Medical Research Council, 1971). However, in the case of this patient, the IgG level measured in 1967 was found to be normal.

In 1963, before the commencement of steroid therapy, a diagnosis of lupus erythematosus was 
seriously considered. Although the biopsy of a small subcutaneous nodule showed evidence of vasculitis, LE cells and antinuclear factor were repeatedly negative. Although neutropenia is a frequent finding in untreated lupus (discoid and systemic), Dubois warns against the acceptance of severe degrees of neutropenia as being causally related to systemic lupus, only $5 \%$ of his series of 500 cases having total white counts lower than 3000 and less than $1 \%$ below 2000 (Dubois, 1966). The possible role of hypersplenism has not been discounted in the neutropenia of systemic lupus, a possibility obviously not applicable in this case. Although leucocyte agglutinins were not looked for in this patient, Killmann (1957) found no correlation between the presence of these antibodies and the occurrence of neutropenia in 40 cases with systemic lupus erythematosus, rheumatoid arthritis, and various other collagen disorders.

The episode in 1963, during which she developed persistent fever and muscle wasting in association with neutropenia, which responded remarkably to steroid therapy both symptomatically and with recovery of her depressed neutrophil levels, is somewhat puzzling, but would be consistent with an underlying collagen disorder. Steroid therapy is known to increase the release of neutrophils into the blood stream and to inhibit their movement out of it, thus raising circulating neutrophil levels (Bishop, Athens, Boggs, Warner, Cartwright, and Wintrobe, 1968). However, the degree to which her neutrophil count apparently responded far exceeded the usual size of the response to pharmacological doses of steroids in normal individuals, and is as far as we are aware, unreported in spontaneous, cyclic, and familial neutropenia.

The finding of extensive small-vessel amyloidosis contrasted with the lack of any evidence of arteritis at necropsy. The coincident anatomical distribution of these two processes is unlikely to have been fortuitous, and it seems reasonable to deduce that the small vessel arteritis progressed to small vessel amyloidosis. Such a relationship has previously been described (Heptinstall, Porter, and Barkley, 1954); of course the relationship of amyloidosis to collagen disorders in general is well known (Jennings, 1950; Dubois, 1966). Perhaps the repeated stimulation of the reticuloendothelial system in a patient with neutropenia led eventually to a breakdown in autoregulation and the consequent development of an incompletely characterized collagen disorder.

We are grateful to Dr L. Kreel for performing the arteriogram and to Drs G. Slavin and A. Darby for preparing and commenting on the histological sections.

\section{References}

Bishop, C. R., Athens, J. W., Boggs, D. R., Warner, H. R., Cartwright, G. E., and Wintrobe, M. M. (1968). Leukokinetic studies. XIII. A non-steady-state kinetic evaluation of the mechanism of cortisone-induced granulocytosis. J. clin. Invest., 47, 249-260.

Boggs, D. R., and Wintrobe, M. M. (1970). Alterations in leukocytes. In Harrison's Principles of Internal Medicine, 6th ed., edited by M. M. Wintrobe et al, pp. 329-338. McGraw-Hill, New York.

Dubois, E. L. (1966). The clinical picture of systemic lupus erythematosus. In Lupus Erythematosus, edited by E. L. Dubois, pp. 129-276. McGraw-Hill, New York.

Heptinstall, R. H., Porter, K. A., and Barkley, H. (1954). Giant-cell (temporal) arteritis. J. Path. Bact., 67, 507-519.

Jennings, G. H. (1950). Amyloidosis in rheumatoid arthritis. Brit. med. J., 1, 753-756.

Killmann, S. A. (1957). Leukocyte agglutinins in collagen disease. Acta rheumat. scand., 3, 209-227.

Medical Research Council Working Party on Hypogammaglobulinaemia (1971). Hypogammaglobulinaemia in the United Kingdom. Spec. Rep. Ser. Med. Res. Coun., 310. HMSO, London.

Morley, A. A., Carew, J. P., and Baikie, A. G. (1967). Familial cyclical neutropenia. Brit. J. Haemat., 13, 719-738.

Reimann, H. A. (1963). Periodic Diseases. Blackwell, Oxford. 